\title{
Atrial Tachycardia with Multiple Reconductions Across the Surgical Incision
}

\author{
Takayuki Nagai ${ }^{1}$, Hiroshi Kawakami ${ }^{1}$, Yasuhiro Sasaki ${ }^{1}$, Akira Fujii ${ }^{1}$, Katsuji Inoue ${ }^{1}$, \\ Shuntaro Ikeda ${ }^{1}$, and Osamu Yamaguchi ${ }^{1}$ \\ ${ }^{1}$ Ehime University Graduate School of Medicine
}

May 27, 2020

\begin{abstract}
Incisional atrial tachycardia (AT) with multiple penetrating points on one surgical incision has not been reported. We present a case of incisional AT following mitral annuloplasty with a superior transseptal approach, in which two reconduction sites were part of the reentrant circuit. Radiofrequency ablation at the reconduction site successfully terminated the tachycardia. A total of four penetrating points were found on the incision line, and radiofrequency ablation at these sites was completed. Detailed mapping with consideration of possible reconduction sites along the incision line should be performed to avoid further instances of AT following open heart surgery.
\end{abstract}

\section{Case report}

A 54-year-old man was referred to our hospital because of palpitation attacks. He had a history of mitral annuloplasty with a superior transseptal (STS) approach for mitral valve prolapse at the age of 49 years. Atrial flutter was recognized by 12-lead echocardiography (ECG); thus, catheter ablation was performed. When both atria were mapped using the CARTO3 system (Biosense Webster, Diamond Bar, CA) during tachycardia, double potentials, indicating an incision line connecting the right atrial free wall to the septum and then to the left atrial roof, were observed (Figure 1A ). The typical counterclockwise atrial flutter with a cycle length of $260 \mathrm{~ms}$ was mapped and terminated during cavotricuspid isthmus (CTI) ablation. Subsequently, atrial tachycardia (AT) with a cycle length of $272 \mathrm{~ms}$ was induced. The entrainment and activation mapping revealed a macroreentrant circuit associated with the right atrial part of the incision line from the STS approach. The activation wavefront circulated clockwise around the incision with two penetration points along the surgical incision in right atrium (Figure $1 B$ ). AT was rapidly terminated after 2 seconds of the first radiofrequency application $(30 \mathrm{~W}, 60$ seconds in total) at the conduction site across the incision of the right atrial free wall. AT was not induced thereafter. Another conduction point across the incision was also ablated. In addition, a linear ablation connecting the incisional scar with the inferior vena cava (IVC) was created. Then, pacing from the low lateral right atrium (anterior side of the incision line) showed residual conduction beyond surgical incision at 10 O'clock and 11 O'clock positions viewing the annulus as a clockface (Figure 2, $A$ and $B$ ). Radiofrequency application at these sites was performed, and complete conduction block was verified (Figure 2, $C$ ). The excitation of sinus rhythm propagates behind the incision, then turns to the tricuspid annulus (anterior side of the incision) and ascends in a counterclockwise manner to the free wall. The two block lines (incision to IVC and CTI) make the posterolateral wall of the tricuspid annulus the most delayed site of propagation during sinus rhythm in the right atrium. The patient has been free of recurrence of tachycardia for three years after ablation.

\section{Discussion}

Intra-atrial reentrant tachycardia is a well-known complication after open heart surgery with atriotomy. 
1-4 Typical atrial flutter is the most commonly presenting arrhythmia in this patient population, often combined with incisional AT associated with postoperative scars due to atriotomy and/or cannulation. ${ }^{1,2}$ The STS approach provides excellent exposure for mitral valve reconstruction. However, this method may be proarrhythmic compared with the other left atrial approach because the it involves an extensive series of incisions. ${ }^{3}$ The incision line acts as the central obstacle during incisional AT, and a postoperative myocardial conduction delay between the incision line and other structures (tricuspid valve and/or IVC) is believed to facilitate the formation of the reentrant circuit after this approach. ${ }^{4}$ Therefore, typical atrial flutter is the most frequent circuit of postoperative AT in this patient population. Incisional AT, which develops around the right atrial part of the incision by the STS approach, is likely to occur subsequently. In the present case, four resumption points across the surgical incision were observed; however, two penetrations were involved in the tachycardia circuit. The other two areas between these points are likely to present functional conduction block during tachycardia (Figure 3 ). Although the precise mechanism of electrical propagation across a theoretically inexcitable surgical suture line is unknown, three possible mechanisms (mechanical coupling, electronic influence, and direct electrical conduction) are proposed. ${ }^{5}$ It is certain that conduction across the contralateral side of the incision line can occur, as shown by studies regarding AT between the donor and recipient hearts after heart transplantation. ${ }^{6-8}$ It is reasonable to consider that the same phenomenon can occur in an autologous heart. In a case series of 16 patients by Lukac et al., ${ }^{4}$ the activation wavefront circulating around the entire right atrial part of the incision was confirmed in all but one patient with incisional AT. In this paper, incisional AT that revolved around the incision line with one penetration point was shown. However, incisional AT with multiple penetrating points on one surgical incision has not been reported so far.

The recurrence rate after AT ablation following open heart surgery is relatively high (20\%-25\%). ${ }^{9,10}$ Kanagasundram et al. reported the importance of complete block from the lateral incision to IVC of the right atrium to prevent recurrence after ablation of incisional AT following the STS approach. ${ }^{11}$ It is also important to assess the presence or absence of reconduction along the incision line, and if present, additional radiofrequency application may help prevent future AT recurrences.

\section{Conclusion}

In patients with AT after open heart surgery, there may be sites of reconduction across the atrial incision line, which might lead to further instances of ATs, and detailed mapping and catheter ablation based on this perspective should be conducted.

\section{References}

1. Anné W, Van Rensburg H, Adams J, Ector H, Van de Werf F, Heidbüchel H. Ablation of postsurgical intra-atrial reentrant tachycardia. Predilection target sites and mapping approach. Eur Heart J 2002;23:1609-1616.

2. Roca-Luque I, Rivas Gándara N, Dos Subirà L, et al. Intra-atrial re-entrant tachycardia in congenital heart disease: types and relation of isthmus to atrial voltage. Europace 2018;20:353-361.

3. Lukac P, Hjortdal VE, Pedersen AK, Mortensen PT, Jensen HK, Hansen PS. Atrial incision affects the incidence of atrial tachycardia after mitral valve surgery. Ann Thorac Surg 2006;81:509-513.

4. Lukac P, Hjortdal V, Pedersen AK, Jensen HK, Mortensen PT, Hansen PS. The superior transseptal surgical approach to mitral valve creates slow conduction. Pacing Clin Electrophysiol 2006;29:719-726.

5. Birnie D, Green MS, Veinot JP, Tang AS, Davies RA. Interatrial conduction of atrial tachycardia in heart transplant recipients: potential pathophysiology. J Heart Lung Transplant 2000;19:1007-1010.

6. Nof E, Stevenson WG, Epstein LM, Tedrow UB, Koplan BA. Catheter ablation of atrial arrhythmias after cardiac transplantation: Findings at EP study utility of 3-D mapping and outcomes. J Cardiovasc Electrophysiol 2013;24:498-502.

7. Lau EW, Birnie DH. One heart, two minds. Europace 2005;7:535-536.

8. Saoudi N, Redonnet M, Anselme F, Poty H, Cribier A. Catheter ablation of atrioatrial conduction as a cure for atrial arrhythmia after orthotopic heart transplantation. J Am Coll Cardiol 1998;32:1048-1055.

9. Anguera I, Dallaglio P, Macías R, et al. Long-Term outcome after ablation of right atrial tachyarrhyth- 
mias after the surgical repair of congenital and acquired heart disease. Am J Cardiol 2015;115:17051713.

10. Roca-Luque I, Rivas-Gándara N, Dos Subirà L, et al. Long-term follow-up after ablation of intra-atrial re-entrant tachycardia in patients with congenital heart disease: types and predictors of recurrence. JACC Clin Electrophysiol 2018;4:771-780.

11. Kanagasundram AN, Baduashvili A, Liu CF, et al. A novel criterion for conduction block after catheter ablation of right atrial tachycardia after mitral valve surgery. Circ Arrhythm Electrophysiol 2013;6:3947.

\section{Figure Legends}

\section{Figure 1.}

A: A three-dimensional electroanatomical biatrial activation map (CARTO3) during typical atrial flutter. The activation wavefront is found to revolve in a counterclockwise manner around the TA. The atriotomy of superior transseptal approach line (blue dots) extends from the lateral wall of the right atrium through the septum to the roof of the left atrium. The brown dots indicate ablation points. B: An activation map of the right atrium during AT, which was induced after cavotricuspid isthmus ablation. The activation wavefront rotates the right atrial part of the incision line in a clockwise manner through the two penetration points of the incision. AT was rapidly terminated after the first radiofrequency application at conduction site (white asterisk) of the right atrial free wall.

The yellow line indicates the superior transseptal incision. The brown dots indicate ablation points. White circles indicate possible sites of IVC cannulation.

AT, atrial tachycardia; IVC, inferior vena cava; TA, tricuspid annulus; MA, mitral annulus.

\section{Figure 2.}

A, B: An activation sequence at the posterior side of the incision line during pacing from the low lateral right atrium (white arrow, opposite side of the incision) following successful cavotricuspid isthmus and subsequent incisional AT ablation. The conduction site between the lower end of the incision and the IVC has been also ablated. The residual conduction site beyond the surgical incision at the 10 O'clock position viewing the annulus as a clockface was revealed (A, black asterisk). After radiofrequency application in this area, another conduction site at 11 O'clock emerged (B, orange asterisk). Note the local bipolar electrograms before and after ablation at each ablation site showing marked local electrogram delay from the pacing spike.C: The right atrial activation sequence during sinus rhythm after all ablation procedures. No residual conduction across the surgical incision was confirmed. The yellow line indicates the superior transseptal incision. The brown and pink dots indicate ablation points.

AT, atrial tachycardia; IVC, inferior vena cava; SN, sinus node; TA, tricuspid annulus.

\section{Figure 3 .}

A schematic diagram illustrating distribution of four resumption sites across the surgical incision. The red line shows the incision. The blue arrows indicate activation of the re-entrant circuit of incisional AT (A, B ) and activation sequence of the lateral right atrium during pacing from the low lateral right atrium $(\mathbf{C}, \mathbf{D}$ ). Numbers in circle indicate the sequence and location of ablation of the reconduction sites on the incision. Sites of ablation are indicated by pink dots. Note that two penetration points (circled number 3 and 4 indicated by short solid orange line) might demonstrate functional conduction block during incisional AT.

AT, atrial tachycardia.

Figures 

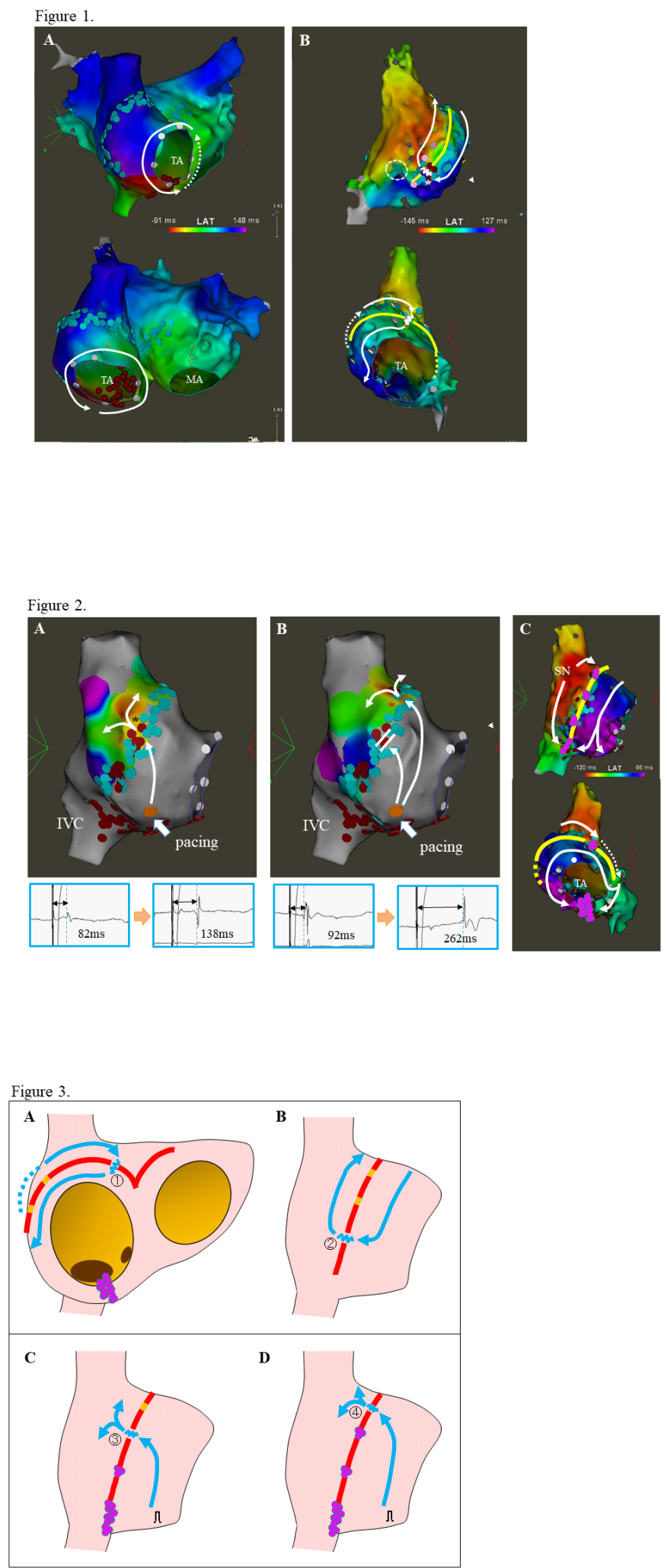